\title{
Optimale Eigenfinanzierung der Personenunternehmen nach der Unternehmensteuerreform 2008/2009
}

\author{
StB Prof. Dr. Stefan Homburg*, Dipl.-Kffr. Henriette Houben ${ }^{* *}$ \\ and Prof. Dr. Ralf Maiterth ${ }^{* *}$
}

Discussion Paper No. 365

May 2007

ISSN: 0949-9962

\begin{abstract}
Effective from 2008, Germany's top marginal income tax rate rises from 42 to 45 per cent. However, sole proprietorships and partnerships can opt for a preferential tax rate of 28.25 per cent for retained profits ( $\$ 34 \mathrm{a}$ EStG-E). If they do so, profits are taxed again upon distribution. Using corporate finance tools, we analyse in detail whether or not the option is favourable. However, these tools do not suffice since the new tax regime is too complicated. Costs of capital cannot be calculated explicitly any more, but we derive exact implicit characterizations. Concrete numerical examples are also provided.
\end{abstract}

JEL-Classification: H25, M10, D92

Keywords: Business Taxes, German Income Tax

\footnotetext{
* Leibniz University Hannover, School of Economics and Management, Institute of Public Finance.

${ }^{* *}$ Leibniz University Hannover, School of Economics and Management, Institute of Business Taxation.
} 


\section{Einleitung}

Seit gut 40 Jahren untersuchen Steuerlehre und Finanzierungstheorie den Einfluss der Besteuerung auf die Finanzierungsentscheidungen der Kapitalgesellschaften. Als Stichworte seien Harbergers Kritik der klassischen Körperschaftsteuer genannt ${ }^{1}$, der Disput zwischen „Old View“ und „New View“2 oder der WACC-Ansatz ${ }^{3}$. Die Disziplin „corporate finance" gilt als so diffizil, dass viele Steuerberatungsgesellschaften hierfür eigene Kompetenzzentren unterhalten. Demgegenüber haben sowohl die Steuerlehre als auch die Finanzierungstheorie der Finanzierung von Personenunternehmen bisher keine vergleichbare Aufmerksamkeit geschenkt, und zwar mit Recht. Unternehmer, die bereit waren, über kleinere Unebenheiten wie Gewerbesteuerüberhänge oder den Sparerfreibetrag hinwegzusehen, konnten ihre Finanzierung ohne Rücksicht auf Steuern wählen, weil alle Finanzierungswege ähnliche Belastungen auslösten. ${ }^{4}$

Nach Inkrafttreten der Unternehmensteuerreform 2008/2009 wird das anders sein, und zwar aus zwei Gründen: Erstens werden Einkünfte aus Kapitalvermögen gemäß $₫ 32 \mathrm{~d}$ EStG-E grundsätzlich nicht mehr der tariflichen Einkommensteuer unterworfen, sondern der meist niedrigeren Abgeltungsteuer. ${ }^{5}$ Zweitens können (Einzel- und Mit-) Unternehmer für nicht entnommene Gewinne gemäß $\$ 34 \mathrm{a}$ EStG-E eine begünstigte Besteuerung beantragen, die bei späterer Entnahme eine Nachversteuerung auslöst. ${ }^{6}$ Die optimale Finanzierung eines Personenunternehmens hängt von insgesamt vier Steuern ab, deren Belastungswirkungen sich im Allgemeinen der Höhe und dem Zeitpunkt nach unterscheiden. Insgesamt hat der Gesetzgeber ein System, das für Personenunternehmen tendenziell Finanzierungsneutralität sicherte, durch ein stark verzerrendes Schedulensystem ersetzt, das neuartige Fragen aufwirft. ${ }^{7}$

Eine dieser Fragen, nämlich die nach der steuerlich günstigsten Finanzierung, ist schon geklärt, und die Antwort kann auch nicht verwundern: In Abwesenheit außersteuerlicher Beschränkungen sollten Unternehmer stets zur Fremdfinanzierung greifen, weil alle Formen der Eigenfinanzierung stärker belastet werden. ${ }^{8}$ Dies stimmt jedenfalls unter der

1 Harberger (1962).

2 Vgl. zur Diskussion Sinn (1990); Sorensen (1995).

3 Vgl. zum WACC-Ansatz Kruschwitz/Löffler (2006), S. 69-71 oder Kruschwitz (2007), S. 410-414.

4 Die Finanzierungswege vergleichende Beispielrechnungen finden sich bspw. in Schreiber (2002), S. 558-560; Spengel (2006), S. G26; Maiterth/Sureth (2006), S. 239-242 und in Sachverständigenrat (2006), S. 169.

5 Zur Wirkung einer Abgeltungsteuer siehe Kiesewetter/Lachmund (2004).

6 Zum Zusammenspiel von Abgeltungsteuer und Begünstigung einbehaltener Gewinne in Österreich vgl. Kiesewetter/Niemann (2004).

7 Bei Unsicherheit oder aneutraler Gewinnermittlung konnte auch das bisherige Steuerregime keine Finanzierungsneutralität sichern. Diese beiden Aspekte werden im hiesigen Text vernachlässigt, um den Blick auf das Wesentliche zu lenken. Zu investitionsneutralen Steuersystemen unter Sicherheit vgl. König (1997) und zu solchen unter Unsicherheit Sureth (1999); Niemann (2001); Löffler/Schneider (2003).

8 Hierzu Homburg/Houben/Maiterth (2007). 
typischerweise erfüllten Voraussetzung, dass der tarifliche Einkommensteuersatz den Satz der Abgeltungsteuer überschreitet. Die Fremdfinanzierung ist in diesem Fall nicht nur der Eigenfinanzierung bei regulärer Versteuerung nach $\$ 32 \mathrm{a}$ EStG überlegen, sondern auch der neuen begünstigten Versteuerung nach $\$ 34 \mathrm{a}$ EStG-E, weil Gewinne bei Inanspruchnahme der Begünstigung doppelt besteuert werden und schon allein der begünstigte Einkommensteuersatz gemäß $\$ 34 \mathrm{a}$ EStG-E $(28,25 \%)$ den Abgeltungsteuersatz gemäß $\$ 32 \mathrm{~d}$ EStG-E (25\%) übersteigt.

Gleichwohl werden manche Personenunternehmen ihre Investitionen auch künftig durch einbehaltene Gewinne oder Einlagen finanzieren, obwohl sie dadurch steuerliche Nachteile erleiden. Hierbei lassen sich zwei Motive unterscheiden: Erstens kann es gesellschaftsvertragliche Entnahmebeschränkungen geben. Zweitens unterliegen Personenunternehmen bisweilen einer Kreditrationierung; dies gilt insbesondere für forschungsorientierte Unternehmen, die keine geeigneten Sicherheiten bieten können. In Anwesenheit solcher Beschränkungen stellt sich die Frage, ob einbehaltene Gewinne gemäß $\$ 34 \mathrm{a}$ EStG-E begünstigt versteuert werden sollten und welche Auswirkungen von der optimalen Steuerpolitik auf die Kapitalkosten der Eigenfinanzierung ausgehen.

Die Arbeit ist wie folgt aufgebaut: In Abschnitt 2 werden die durch die neue Rechtslage definierten entscheidungsrelevanten Steuersätze hergeleitet. Sie betreffen die Belastung normal und begünstigt versteuerter Gewinne, die Nachversteuerung sowie die Belastung von im Privatvermögen gehaltenen Finanzanlagen. Abschnitt 3 analysiert sodann, inwieweit die Stellung eines Antrag auf begünstigte Besteuerung einbehaltener Gewinne optimal ist. Aufbauend auf diesen Ergebnissen werden in Abschnitt 4 die Kapitalkosten der Selbstfinanzierung der Personenunternehmen berechnet, die sich bei optimaler Antragstellung ergeben. Dasselbe geschieht in Abschnitt 5 analog für die Kapitalkosten der Beteiligungsfinanzierung. In Abschnitt 6 wird gezeigt, unter welchen Voraussetzungen es optimal ist, den Antrag auf begünstigte Besteuerung für den Gesamtgewinn zu stellen, obwohl hierbei ständige Zuschüsse aus dem Privatvermögen geleistet werden müssen, um die fälligen Steuern zu begleichen. Alle Resultate werden in Abschnitt 7 durch konkrete Zahlenbeispiele illustriert, und Abschnitt 8 beschließt die Arbeit.

\section{Steuersätze und Steuerfaktoren}

Wie schon bemerkt, kommt die Analyse der Besteuerung von Personenunternehmen künftig nicht mehr mit einem Steuersatz aus, sondern erfordert die Unterscheidung vieler verschiedener Steuersätze. Diese seien zunächst unter Beachtung der neuen Rechtslage hergeleitet und gleichzeitig zu möglichst wenigen kompakten Sätzen gebündelt, um die spätere Analyse einfach und übersichtlich zu halten. Dabei sind nominale und effektive Steuersätze zu unterscheiden. Als effektive Steuersätze werden diejenigen Steuersätze bezeichnet, die sich auf den Gewinn vor Steuern beziehen, während die steuerrechtlich definierte Bemessungsgrundlage Bezugsgröße für die nominalen Steuersätze ist. 
Begonnen wird mit dem Satz s $\mathrm{e}^{\mathrm{e}}$, der die Gesamtbelastung eines nicht gewerbesteuerpflichtigen Unternehmers durch die tarifliche Einkommensteuer gemäß $₫ 32 \mathrm{a}$ EStG mit dem Satz s ${ }^{\text {tarif }}$, den Solidaritätszuschlag und gegebenenfalls die Kirchensteuer mit dem Satz k zusammenfasst: ${ }^{9}$

$$
s^{\mathrm{e}}=\frac{\mathrm{s}^{\mathrm{tarif}}}{1+\mathrm{k} \cdot \mathrm{s}^{\text {tarif }}} \cdot(1,055+\mathrm{k}) .
$$

Bei Gewerbesteuerpflicht unterliegt der Gewinn außerdem einer Belastung von

$$
s^{\mathrm{g}}=\mathrm{H} \cdot 0,035-\min \{\mathrm{H} \cdot 0,035 ; 3,8 \cdot 0,035\} \cdot 1,055 .
$$

Mit $\mathrm{H}$ als Gewerbesteuer-Hebesatz repräsentiert $s^{\mathrm{g}}$ die effektive Gewerbesteuerbelastung unter Berücksichtigung der auf einen Hebesatz von $380 \%$ beschränkten Anrechnung gemäß $₫ 35$ EStG-E auf die tarifliche Einkommensteuer.

Einbehaltene Gewinne werden entweder mit $s^{\mathrm{e}}+s^{\mathrm{g}}$ besteuert ${ }^{10}$ oder, bei Stellung des Antrags auf begünstigte Besteuerung nach $\$ 34 \mathrm{a} E S t G-E$, mit $s^{\mathrm{b}}+s^{\mathrm{g}}$, wobei sich der nominale begünstigte Steuersatz $s^{\mathrm{b}}$ einschließlich Solidaritätszuschlag und Kirchensteuer wie folgt errechnet ${ }^{11}$ :

$$
s^{\mathrm{b}}=\frac{0,2825}{1+\mathrm{k} \cdot \mathrm{s}^{\mathrm{tarif}}} \cdot(1,055+\mathrm{k})
$$

Eine spätere Entnahme begünstigt besteuerter Gewinne löst eine Nachversteuerung $(\$ 34$ a Abs. 4 EStG-E) mit dem folgenden Nominalsteuersatz aus:

$$
s^{\mathrm{nv}}=\frac{0,25}{1+\mathrm{k} \cdot \mathrm{s}^{\mathrm{tarif}}} \cdot(1,055+\mathrm{k}) .
$$

Einkünfte aus Kapitalvermögen unterliegen im Rahmen der Abgeltungsteuer $(\$ 32 \mathrm{~d}$ EStG-E) einer nominalen Belastung mit Einkommen- und Kirchensteuer von

$$
\mathrm{s}^{\mathrm{a}}=\frac{0,25}{1+\mathrm{k} \cdot 0,25} \cdot(1,055+\mathrm{k})
$$

9 Der Steuersatz aus Gleichung (1) entspricht auch der Tarifbelastung anderer Einkunftsarten, die der progressiven Einkommensteuer unterliegen.

10 Die Gewerbesteuer ist gemäß $₫ 4$ Abs. 5b EStG keine Betriebsausgabe mehr. Teile der Literatur sprechen von einer nicht abziehbaren Betriebsausgabe, so als hätte der Gesetzgeber die Gewerbesteuer in $\$ 4$ Abs. 5 EStG eingereiht (Förster (2007), S. 763; Kleineidam/Liebchen (2007), S. 409; Kessler/Ortmann-Babel/Zipfel (2007), S. 526). Tatsächlich wird die Gewerbesteuer nicht außerbilanziell hinzugerechnet, sondern bereits beim Übergang von der Handelsbilanz zur Steuerbilanz addiert. Für Unternehmer ist das vorteilhaft, weil die begünstigte Besteuerung an den Gewinn laut Steuerbilanz anknüpft.

11 Voraussetzung für die hier modellierte Entlastungswirkung des Sonderausgabenabzugs der Kirchensteuer ist die Existenz weiterer Einkünfte, die dem tariflichen Einkommensteuersatz $\mathrm{s}^{\text {tarif }}$ unterliegen. Andernfalls wird die Entlastung infolge des Sonderausgabenabzugs i.d.R. durch $s^{\mathrm{b}}$ determiniert. 
Der Einkommensteuersatz bei Nachversteuerung und der nominale Abgeltungsteuersatz lt. $\$ 32$ d EStG-E sind zwar identisch, nämlich gleich $25 \%$, doch unterscheiden sich die Nominalbelastungen im Fall der Kirchensteuerpflicht regelmäßig, weil die Kirchensteuer im ersten Fall als Sonderausgabe die Bemessungsgrundlage der tariflichen Einkommensteuer mindert, im letzteren Fall aber die Bemessungsgrundlage der Abgeltungsteuer selbst (\$ 32d Abs. 1 S. 3-5 EStG-E i. V. m. \$ 10 Abs. 1 Nr. 4 EStG).

Um die folgenden Herleitungen übersichtlich zu halten, erweist es sich als zweckmäßig, nicht mit Steuersätzen zu arbeiten, sondern mit den wie folgt definierten Steuerfaktoren:

$$
\mathrm{E}=1-s^{\mathrm{e}}-\mathrm{s}^{\mathrm{g}} \text { und } \mathrm{A}=1-\mathrm{s}^{\mathrm{a}} .
$$

Der Steuerfaktor E entspricht dem Bruchteil des Gewinns vor Steuern, der dem Unternehmer im Fall der regulären Besteuerung nach $\$ 32 \mathrm{a}$ EStG nach Abzug von Einkommensteuer und Solidaritätszuschlag sowie etwaiger Gewerbesteuer und Kirchensteuer unter Berücksichtigung der Gewerbesteueranrechnung und des Abzugs der Kirchensteuer als Sonderausgabe verbleibt. Analog repräsentiert A den nach Steuern verbleibenden Bruchteil von Einkünften aus Kapitalvermögen.

Bei begünstigter Versteuerung einbehaltener Gewinne gemäß $₫ 34 a$ Abs. 1 EStG-E kann der Unternehmer, ohne zusätzliche Mittel aus dem Privatvermögen zu verwenden, den Begünstigungsantrag nicht für den Gesamtgewinn stellen, selbst wenn er auf Entnahmen für Konsumzwecke völlig verzichtet, weil er einen Teil des Gewinns für Steuerzahlungen entnehmen muss. ${ }^{12}$ Nur bei Entnahme der Steuerzahlung ist der Unternehmer so gestellt wie der Anteilseigner einer Kapitalgesellschaft im Vollthesaurierungsfall, der während der Thesaurierungsphase keine Zuschüsse aus seinem Privatvermögen leistet. Mit B als dem Anteil des Gewinns, der nach Zahlung der Steuern in die Rücklage eingestellt wird, erhält man durch Abzug der Steuern den Steuerfaktor $B=1-s^{\mathrm{g}}-s^{\mathrm{b}} \cdot \mathrm{B}-s^{\mathrm{e}} \cdot(1-\mathrm{B})$, denn die Entnahme 1-B unterliegt der gewöhnlichen Gewinnbelastung. ${ }^{13}$ Durch Auflösen folgt

$$
\mathrm{B}=\frac{1-s^{\mathrm{e}}-s^{\mathrm{g}}}{1-s^{\mathrm{e}}+s^{\mathrm{b}}}
$$

Der Steuerfaktor B gibt mithin an, welchen Anteil des Gewinns der Unternehmer höchstens einbehalten kann, wenn er bei unberührtem Privatvermögen auf Entnahmen für private Konsumzwecke verzichtet. Bei Konfessionslosigkeit, einem Gewerbesteuerhebesatz von $400 \%$ und einer tariflichen Belastung von $42 \%$ errechnet man für B den Wert 65,2 \%. Die effektive Steuerbelastung einbehaltener Gewinne beträgt damit 34,8\% und nicht, wie oft kolportiert, 29,8\%. Sie liegt rund fünf Punkte über der Belastung thesaurierter Gewinne einer Kapitalgesellschaft. Schon deshalb ist der jargonhafte Begriff „Thesaurierungsbegünstigung", der eine ähnlich hohe Belastung suggeriert, irreführend.

12 Vgl. Homburg/Houben/Maiterth (2007), S 378-379; Kleineidam/Liebchen (2007), S. 410.

13 Siehe dazu ausführlich Homburg (2007a), S. 688. 
Hat der Unternehmer einen bestimmten Begünstigungsbetrag in Anspruch genommen, verbleibt ihm hiervon im Anschluss an die Nachversteuerung der Bruchteil

$$
\mathrm{N}=1-(1-0,2825 \cdot 1,055) \cdot \mathrm{s}^{\mathrm{nv}} \text {. }
$$

Man beachte, dass der Steuersatz sv ${ }^{\mathrm{nv}}$ gemäß $\$ 34 \mathrm{a}$ Abs. 3 EStG-E nicht mit dem Begünstigungsbetrag multipliziert, sondern der letztere zuvor um die darauf entfallende Steuer und den darauf entfallenden Solidaritätszuschlag, zusammen $29,8 \%$, gekürzt wird. ${ }^{14}$ Die gekürzte Bemessungsgrundlage bezeichnet $\$ 34 \mathrm{a}$ Abs. 3 EStG-E als Nachversteuerungsbetrag.

Der folgende Text nutzt oft die Ungleichung E $>$ N.B. Sie ist bei beliebiger Tarifbelastung und für alle Kirchensteuersätze und Gewerbesteuer-Hebesätze erfüllt und besagt, dass die normale Gewinnbelastung unter der kombinierten Belastung durch begünstigte Versteuerung und Nachversteuerung liegt. Bei einem Gewerbesteuer-Hebesatz von $400 \%$ und Konfessionslosigkeit beträgt die kombinierte Belastung 52,0 \%. Deshalb kann die Stellung eines Antrags auf begünstigte Besteuerung gemäß $\$ 34 \mathrm{a} E S t G-E$ nur aufgrund von Steueraufschubwirkungen vorteilhaft sein.

\section{Antragspolitik für einbehaltene Gewinne}

\subsection{Antragspolitik als Synonym für die Wahlrechtsausübung gemäß $\$ 34 a$ EStG-E}

Nachfolgend wird ein Unternehmer betrachtet, der über die Jahre 0 bis $\mathrm{n}>0$ plant. Der Planungshorizont mag auf der Absicht des Unternehmers beruhen, Gewinne im Anschluss an das Jahr n zu entnehmen, das Unternehmen zu veräußern oder es in eine Kapitalgesellschaft einzubringen; denn alle diese Ereignisse lösen eine Nachversteuerung aus, vgl. $\$ 34$ a Abs. 4 und 6 EStG-E. Das Ziel des Unternehmers besteht darin, das am Anfang des Jahres $\mathrm{n}+1$ vorhandene Vermögen nach Steuern (Endvermögen) zu maximieren. Hierbei ist der Einbehalt des Gewinns vorgegeben, und das einzige Entscheidungsproblem besteht in der Formulierung des Antrags nach $\$ 34 \mathrm{a}$ EStG-E. Der Unternehmer wählt in jedem Jahr eine Zahl $\alpha_{t}$ aus dem Intervall [0; B], die den begünstigt versteuerten Anteil des Gewinns vor Steuern bezeichnet. Folglich bedeutet $\alpha_{\mathrm{t}}=0$ den Verzicht auf die Antragsstellung, und $\alpha_{\mathrm{t}}=\mathrm{B}$ bedeutet den bei konstantem Privatvermögen maximal erreichbaren Antrag. Der Vektor $\left(\alpha_{0}, \ldots, \alpha_{n}\right)$ heißt Antragspolitik.

\subsection{Konstante Antragspolitik}

Bei konstanter Antragspolitik stellt der Unternehmer in jeder Periode den gleichen Antrag: $\alpha_{\mathrm{t}}=\alpha$ für alle Perioden t. Stellt der Unternehmer den Antrag niemals, so gilt $\alpha=0$. Stellt er ihn in jedem Jahr für den bei konstantem Privatvermögen maximal begünsti-

14 Dies verkennen z. B. Kleineidam/Liebchen (2007) oder Kaminski/Hofmann/Kaminskaite (2007), S. 165, die den Nachversteuerungssatz auf den Begünstigungsbetrag statt auf den Nachversteuerungsbetrag anwenden und damit die Regelung in ein zu ungünstiges Licht rücken. 
gungsfähigen Anteil, so gilt $\alpha=\mathrm{B}$. Für alle weiteren Betrachtungen sei der Gewinn der Periode Null auf Eins normiert und eine exogene Unternehmensrendite $\mathrm{r}>0$ unterstellt.

Stellt der Unternehmer den Antrag nach $\$ 34$ a EStG-E niemals, wächst das Endvermögen durch einbehaltene und normal versteuerte Gewinne auf $\mathrm{E} \cdot(1+\mathrm{E} \cdot \mathrm{r})^{\mathrm{n}}$. Dabei ist $\mathrm{E}$ der nach Steuern verbleibende Gewinn aus Periode 0. Dieser wächst in den folgenden Jahren jeweils um den Faktor 1+E·r. Unter der entgegengesetzten Annahme einer Antragsstellung gemäß $\$ 34 \mathrm{a}$ EStG-E in jedem Jahr wächst das Endvermögen auf B· $(1+B \cdot r)^{\mathrm{n}} \cdot \mathrm{N}$. Bei einem tariflichen Steuersatz über 28,25 \% gilt unabhängig von der Gewerbe- und Kirchensteuer B > E, so dass im Fall der Antragsstellung am Ende der Periode 0 eine höhere Rücklage verbleibt, die sich in den folgenden Jahren überdies besser verzinst, nämlich mit dem Faktor 1+B.r. Diese positiven Wirkungen der Antragsstellung sind gegen die in der Multiplikation mit $\mathrm{N}$ zum Ausdruck kommende Nachversteuerung abzuwägen, die das Endvermögen auf gut 80 Prozent der Rücklage kürzt.

Die relative Vorteilhaftigkeit der beiden Antragspolitiken hängt von der Tarifbelastung, der Unternehmensrendite und dem Planungszeitraum ab. Setzt man die beiden Endvermögen gleich und löst nach dem Planungszeitraum auf, so zeigt sich, dass die Antragsstellung vorteilhaft ist, wenn

$$
\mathrm{n}>\frac{\ln (\mathrm{E})-\ln (\mathrm{B} \cdot \mathrm{N})}{\ln (1+\mathrm{B} \cdot \mathrm{r})-\ln (1+\mathrm{E} \cdot \mathrm{r})} .
$$

Der Zähler dieses Bruchs repräsentiert den Nachteil der Antragsstellung, der darin besteht, dass die Normalbelastung des Gewinns stets unter der kumulierten Belastung durch begünstigte Versteuerung und Nachversteuerung liegt, E > B.N. Dieser Nachteil ist ein Einmaleffekt. Ihm gegenüber steht der im Nenner des Bruchs gezeigte Vorteil, dass die Rücklage bei Antragsstellung schneller wächst als bei Antragsverzicht. Anders als der Nachteil der Antragsstellung schlägt dieser Vorteil in jedem Jahr erneut zu Buche. Daher ist eine Antragsstellung in jedem Jahr relativ zum Antragsverzicht in jedem Jahr vorteilhaft, wenn der n-fache Vorteil den einmal auftretenden Nachteil überwiegt.

Die Antragsstellung wird bei hoher Tarifbelastung und hoher Unternehmensrendite früher vorteilhaft. Liegt die Tarifbelastung nicht über 28,25\%, ist die begünstigte Versteuerung immer nachteilig. Hieraus lässt sich aber nicht der Schluss ziehen, die Antragsstellung sei bei tariflichen Belastungen über 28,25 \% vorteilhaft. Ganz im Gegenteil kann die Tarifbelastung, ab der die Antragsstellung lohnt, im Einzelfall weit über 28,25\% liegen, weil die Nachversteuerung erst nach langer Zeit an Bedeutung verliert.

\subsection{Optimale Antragspolitik}

Die bisherigen Ergebnisse beruhen auf der Annahme, der Unternehmer stelle den Antrag auf begünstigte Besteuerung einbehaltener Gewinne entweder in jedem Jahr oder niemals. Das Gesetz sieht eine derartige Bindung nicht vor. Es erlaubt dem Unternehmer vielmehr, den Antrag in einigen Jahren zu stellen und in anderen Jahren zu unterlassen und den Antrag dann, wenn er ihn stellt, auf den maximal erreichbaren Begünstigungsbetrag oder 
nur auf einen Teil davon zu beziehen. Die Menge der Handlungsalternativen ist das aus $\mathrm{n}+1$ Mengen [0; B] gebildete kartesische Produkt $[0 ; \mathrm{B}] \times \ldots \times[0 ; \mathrm{B}]$; die hierdurch beschriebenen Politiken seien gemischte Antragspolitiken genannt.

Weiter oben zeigte sich, dass die Antragsstellung bei wachsendem Planungshorizont tendenziell vorteilhaft wird, weil der Vorteil des dadurch erwirkten Steueraufschubs relativ zum Nachteil der Doppelbesteuerung zunimmt. Dies legt die Vermutung nahe, dass die begünstigte Versteuerung in frühen Jahren eher lohnt als in späten Jahren. Im weiteren Text ist der Antragsverzichtszeitraum a(r) definiert als die kleinste ganze Zahl, für die bei gegebenen Steuerfaktoren gilt:

$$
(\mathrm{B}-\mathrm{E}) \cdot(1+\mathrm{E} \cdot \mathrm{r})^{\mathrm{a}(\mathrm{r})}-\mathrm{B} \cdot(1-\mathrm{N})>0 .
$$

Als Hauptergebnis dieses Beitrags beschreibt der folgende Satz, welche Antragspolitik im neuen System der Unternehmensbesteuerung schlechthin optimal ist.

Satz (Optimale Antragspolitik): Unter der Annahme B > E maximiert der Unternehmer sein Endvermögen, wenn er den Antrag nach $\$ 34 \mathrm{a}$ EStG-E in den Jahren 0 bis $n-\mathrm{a}(\mathrm{r})$ stellt und in den letzten a(r) Jahren nicht stellt.

Beweis: Die Beweisstrategie besteht darin, die optimale Antragspolitik rekursiv zu bestimmen. Dabei ist $i=0 \ldots n$ ein rückwärts laufender Index und $n-i$ der Periodenindex.

i) Sei $\mathrm{i} \leq \mathrm{a}(\mathrm{r})$ : Sofern in den Folgeperioden kein Antrag gestellt wird, wächst das Endvermögen pro Euro Periodengewinn auf B. $(1+E \cdot r){ }^{i}-B \cdot(1-N)$, wenn in Periode $n-i$ ein Antrag auf begünstigte Besteuerung gestellt wird, und sonst auf $\mathrm{E} \cdot(1+\mathrm{E} \cdot \mathrm{r})^{\mathrm{i}}$. Der Vorteil der Antragsstellung, $(\mathrm{B}-\mathrm{E}) \cdot(1+\mathrm{E} \cdot \mathrm{r})^{\mathrm{i}}-\mathrm{B} \cdot(1-\mathrm{N})$, ist laut (10) für $\mathrm{i}<\mathrm{a}(\mathrm{r})$ negativ oder gleich Null. Für $\mathrm{i}=\mathrm{a}(\mathrm{r})$ ist der Vorteil erstmals positiv und wird der Antrag gestellt.

ii) Sei $\mathrm{i}>\mathrm{a}(\mathrm{r})$ : Für $\mathrm{i}=\mathrm{a}(\mathrm{r})+1$ wächst das Endvermögen pro Euro Periodengewinn, wenn der Antrag gestellt wird, auf $\mathrm{B} \cdot(1+\mathrm{B} \cdot \mathrm{r}) \cdot(1+\mathrm{E} \cdot \mathrm{r})^{\mathrm{a}(\mathrm{r})}-\mathrm{B} \cdot(1+\mathrm{B} \cdot \mathrm{r}) \cdot(1-\mathrm{N})$. Andernfalls wächst das

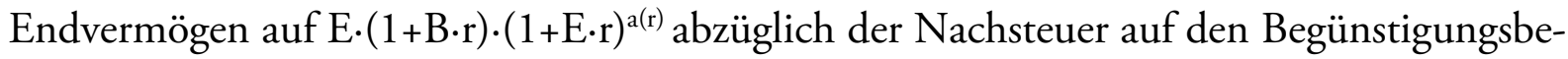
trag aus $\mathrm{i}=\mathrm{a}(\mathrm{r})$. Die letztgenannte Nachversteuerung muss nicht spezifiziert werden, denn ohnehin ist der Vorteil der Antragsstellung größer als

$$
(1+\mathrm{B} \cdot \mathrm{r}) \cdot\left[(\mathrm{B}-\mathrm{E}) \cdot(1+\mathrm{E} \cdot \mathrm{r})^{\mathrm{a}(\mathrm{r})}-\mathrm{B} \cdot(1-\mathrm{N})\right]>0,
$$

wobei die Ungleichung auf (10) beruht. Also wird der Antrag auch für $\mathrm{i}=\mathrm{a}(\mathrm{r})+1$ gestellt. Durch sukzessives Einsetzen größerer i-Werte und Ersatz von $(1+\mathrm{B} \cdot \mathrm{r})$ durch $(1+\mathrm{B} \cdot \mathrm{r})^{\mathrm{i}-\mathrm{a}(\mathrm{r})}$ ersieht man, dass der Antrag in allen früheren Perioden ebenfalls gestellt wird.

iii) Aus der Linearität des Antragsvorteils in Bezug auf den Antragsumfang folgt, dass der Antrag stets in vollem Umfang (B) oder gar nicht gestellt wird.

q.e.d.

Der Satz hat zwei unmittelbare Implikationen. Erstens ist es unter keinen Umständen optimal, den Antrag auf begünstigte Versteuerung in jedem Jahr zu stellen, weil zumindest im Jahre $\mathrm{n}$ der Nachteil der Doppelbesteuerung den Vorteil der Antragsstellung 
überwiegt: Wenn der Unternehmer am Ende des Jahres n den Antrag stellt und den einbehaltenen Gewinn in der nächsten Sekunde, am Anfang des Jahres n+1, entnimmt, verbleibt ihm pro Euro nur der Betrag $\mathrm{B} \cdot \mathrm{N}<\mathrm{E}$, weil die kombinierte Belastung aus begünstigter Versteuerung und Nachversteuerung immer über der regulären Steuerbelastung liegt. Zweitens kann eine Antragsstellung optimal sein, obwohl der Planungszeitraum kürzer ist als der durch Ungleichung (9) beschriebene Zeitraum. Diese Ungleichung beruht nämlich auf der Annahme, dass der Unternehmer den Antrag, sofern er ihn einmal stellt, auch in allen Folgeperioden stellt, während die hier betrachtete gemischte Antragspolitik dies nicht voraussetzt.

Der Antragsverzichtszeitraum a(r) wurde oben als die kleinste ganze Zahl definiert, die der Ungleichung (10) genügt. ${ }^{15} \mathrm{Er}$ ist sogar eine natürliche Zahl, denn nähme man hypothetisch an, dass a(r) verschwindet, wäre (10) wegen $\mathrm{B} \cdot \mathrm{N}<\mathrm{E}$ verletzt. Weil der Ausdruck auf der linken Seite von (10) wegen $\mathrm{B}>\mathrm{E}$ sowohl in $\mathrm{r}$ als auch in $\mathrm{a}(\mathrm{r})$ streng monoton wächst, ist a(r) eine fallende Treppenfunktion. Zur Illustration sei ein Unternehmer betrachtet, der bis zum Jahr $\mathrm{n}=10$ plant und $\mathrm{a}(\mathrm{r})=3$ berechnet hat. Für diesen Unternehmer besteht die optimale Politik darin, den Antrag auf begünstigte Besteuerung in den Jahren 0 bis $7 \mathrm{zu}$ stellen und in den Jahren 8 bis 10 darauf zu verzichten.

Gilt im Einzelfall $\mathrm{a}(\mathrm{r})>\mathrm{n}$, was durchaus vorkommen kann, dann sollte der Unternehmer dem obigen Satz zufolge in allen Perioden auf den Antrag verzichten. Unter dieser Voraussetzung wächst das Endvermögen pro Euro Gewinn der Periode 0 in klassischer Manier auf $\mathrm{E} \cdot(1+\mathrm{E} \cdot \mathrm{r})^{\mathrm{n}}$. Unter der entgegengesetzten Annahme $\mathrm{a}(\mathrm{r}) \leq \mathrm{n}$ lässt sich das Wachstum einer in Periode 0 gebildeten und begünstigt versteuerten Rücklage bis zum Beginn der Periode n+1 durch den folgenden Aufzinsungsfaktor beschreiben:

$$
\Omega(\mathrm{r}, \mathrm{n})=(1+\mathrm{B} \cdot \mathrm{r})^{\mathrm{n}-\mathrm{a}(\mathrm{r})} \cdot\left[(1+\mathrm{E} \cdot \mathrm{r})^{\mathrm{a}(\mathrm{r})}-(1-\mathrm{N})\right] .
$$

Der Aufzinsungsfaktor $\Omega$ zeigt, wie die Rücklage in den ersten Jahren bei begünstigter Versteuerung mit dem Faktor 1+B.r und später, im Antragsverzichtszeitraum, mit dem Faktor 1+E.r wächst. 1-N ist der Nachversteuerungssatz. Bezogen auf einen Euro Gewinn vor Steuern in Periode 0 beträgt das Endvermögen $B \cdot \Omega(r, n)$, wenn der Antrag auf begünstigte Besteuerung mindestens in Periode 0 gestellt wird.

Für spätere Zwecke sei festgehalten, dass der Aufzinsungsfaktor das folgende Grenzverhalten zeigt, wie man durch Potenzieren von (12) mit 1/n sofort erkennt:

$$
\lim _{\mathrm{n} \rightarrow \infty}[\Omega(\mathrm{r}, \mathrm{n})]^{1 / \mathrm{n}}=1+\mathrm{B} \cdot \mathrm{r} .
$$

Die linke Seite der Gleichung ist der Grenzwert des geometrischen Mittels der Zinsfaktoren nach Steuern. Bei unbeschränkt wachsendem Planungszeitraum nähert sich dieses Mittel dem Wert 1+B.r, weil die Nachversteuerung und der Antragsverzichtszeitraum, in dem die Periodenrendite nach Steuern nur 1+E.r beträgt, an Bedeutung verlieren.

15 Durch Auflösen folgt, dass a(r) dem ganzzahligen Wert von $1+[\ln (\mathrm{B} \cdot(1-\mathrm{N}))-\ln (\mathrm{B}-\mathrm{E})] / \ln (1+\mathrm{E} \cdot \mathrm{r})$ entspricht. 


\section{Optimale Selbstfinanzierung}

Die obigen Ergebnisse zur optimalen Antragspolitik beeinflussen die relative Vorteilhaftigkeit der Finanzierungswege eines Personenunternehmens. Dies sei zunächst für die Finanzierung durch einbehaltene Gewinne (Selbstfinanzierung) gezeigt, die vor allem reifen Personenunternehmen offensteht, namentlich traditionellen Familienpersonengesellschaften. Für solche Unternehmen stellt sich die Frage, bis zu welchem Punkt der Einbehalt von Gewinnen gegenüber der Entnahme vorteilhaft ist. Eine ökonomisch fundierte Antwort hierauf erhält man nicht durch die in der Praxis beliebten Belastungsvergleiche, weil ein Vergleich von Steuersätzen in Gegenwart von Steueraufschubeffekten irreführt. Vielmehr ist die Unternehmensrendite - wenn man andere Finanzierungsformen vernachlässigt ${ }^{16}$ - mit den Kapitalkosten der Selbstfinanzierung zu vergleichen. Die letzteren sind definiert als jene Rendite vor Steuern, die das Unternehmen erwirtschaften muss, damit der Gewinneinbehalt dasselbe Endvermögen ergibt wie eine Entnahme mit anschließender Anlage am Kapitalmarkt. ${ }^{17}$ Ein Gewinn vor Steuern in Höhe von einem Euro wächst bei Entnahme und privater Anlage auf das Endvermögen E. $(1+A \cdot i)^{n}$, wobei $i>0$ den Zinssatz symbolisiert und A den in (6) definierten Bruchteil des Zinsertrags, der nach Zahlung von Abgeltungsteuer, Solidaritätszuschlag und Kirchensteuer verbleibt.

Bei optimaler Antragspolitik, wie sie im vorigen Abschnitt beschrieben wurde, sind die Kapitalkosten der Selbstfinanzierung $\mathrm{r}^{\mathrm{s}}$ implizit definiert durch die Übereinstimmung der Endvermögen bei Einbehalt und Entnahme, also durch

$$
\mathrm{B} \cdot \Omega\left(\mathrm{r}^{\mathrm{s}}, \mathrm{n}\right)=\mathrm{E} \cdot(1+\mathrm{A} \cdot \mathrm{i})^{\mathrm{n}},
$$

sofern der Gewinn mindestens einmal begünstigt versteuert wird. Andernfalls betragen die Kapitalkosten der Selbstfinanzierung $r^{s}=A / E \cdot i$, wie man durch Gleichsetzen der Endvermögen $\mathrm{E} \cdot\left(1+\mathrm{E} \cdot \mathrm{r}^{\mathrm{s}}\right)^{\mathrm{n}}$ und $\mathrm{E} \cdot(1+\mathrm{A} \cdot \mathrm{i})^{\mathrm{n}}$ erkennt. Bei Antragsstellung in mindestens einer Periode lassen sich die Kapitalkosten der Selbstfinanzierung nicht explizit berechnen, sondern nur durch nummerische Verfahren bestimmen. Ihre Ermittlung ist knifflig, weil der Antragsverzichtszeitraum a $\left(\mathrm{r}^{\mathrm{s}}\right)$, der in die Bestimmung des Aufzinsungsfaktors eingeht, seinerseits von den Kapitalkosten abhängt. Da der Ausdruck (14) wenig Intuition hergibt, ist es nützlich, das Grenzverhalten der Kapitalkosten zu charakterisieren. Potenziert man die Gleichung (14) mit 1/n, erhält man unter Nutzung von (13) zunächst den Grenzwertausdruck $1+B \cdot r^{s}=1+A \cdot i$ und damit

$$
\lim _{n \rightarrow \infty} r^{s}=\frac{A}{B} \cdot i
$$

\footnotetext{
16 Existieren andere Finanzierungsmöglichkeiten, wählt man diejenige mit den geringsten Kapitalkosten. In diesem Fall entnimmt man den Gewinn, wenn die Kapitalkosten der Selbstfinanzierung größer als die einer anderen zur Verfügung stehenden Finanzierungsform sind. Vgl. hierzu Homburg (2007b), S. 263.

17 Vgl. Homburg (2007b), S. 258.
} 
Offenbar hängen die Kapitalkosten der Selbstfinanzierung auf Dauer allein von den Steuerfaktoren A und B ab. Der Grund hierfür liegt darin, dass die Nachversteuerung als Einmaleffekt in der Grenzbetrachtung keine Rolle spielt; es kommt nur auf die Verzinsung nach Steuern an. Da der begünstigte Steuersatz über dem Abgeltungsteuersatz liegt, ist A > B. Die Kapitalkosten der Selbstfinanzierung übersteigen den Zinssatz also selbst bei unendlichem Planungshorizont. Insofern wirkt das neue System als Investitionsbremse.

Der Antragsverzichtszeitraum strebt im Grenzübergang gegen die Zahl a(A/B.i). Bei unbeschränkt wachsendem Planungshorizont nimmt die Anzahl der Perioden, in denen die begünstigte Besteuerung gewählt wird, also ständig zu, während die Anzahl der Perioden, in denen kein Antrag gestellt wird, von oben beschränkt bleibt. Der Anteil des Antragsverzichtszeitraums am gesamten Planungszeitraum geht daher asymptotisch gegen Null.

Zusammengefasst liegen die Kapitalkosten der Selbstfinanzierung bei optimaler Antragspolitik im halboffenen Intervall (A/B.i; A/E.i]. Die Obergrenze ist relevant, wenn die Antragsstellung in keiner Periode lohnt; der Untergrenze nähern sich die Kapitalkosten bei unbeschränkt wachsendem Planungshorizont.

\section{Optimale Beteiligungsfinanzierung}

Bisher wurde die begünstigte Besteuerung nach $\$ 34 \mathrm{a}$ EStG-E im Zusammenhang mit der Selbstfinanzierung betrachtet. Die Regelung ist aber auch für die Beteiligungsfinanzierung von Bedeutung, weil ein Unternehmer, der seinem Unternehmen Kapital zuführt, in den folgenden Perioden für die darauf entfallenden zusätzlichen Erträge - so er diese nicht entnimmt - die begünstigte Besteuerung beantragen kann. Ist die Antragsstellung vorteilhaft, mindert sie selbstverständlich die Kapitalkosten der Beteiligungsfinanzierung. Andernfalls erfolgt die Besteuerung nach $\$ 32 \mathrm{a}$ EStG, und die Kapitalkosten der Beteiligungsfinanzierung und Selbstfinanzierung stimmen überein.

Bei einer Kapitalgesellschaft versteht man unter Beteiligungsfinanzierung, dass der Anteilseigner seiner Gesellschaft am Anfang der Periode 1 einen Geldbetrag zuführt und diesen nebst Erträgen am Anfang der Periode $\mathrm{n}+1$ ausschüttet, ohne in der Zwischenzeit weitere Gelder zuzuführen oder abzuziehen. ${ }^{18}$ Um dies für Personenunternehmen nachzubilden, sei daran erinnert, dass ein zum Ende der Periode 0 gebildeter Begünstigungsbetrag in Höhe von einem Euro bis zum Anfang der Periode $n+1$ auf $\Omega(r, n)$ wächst. Eine entsprechende Einlage wächst auf $\Omega(r, n)+1-N$, wenn der Unternehmer in den Perioden 1 bis $n$ nichts einlegt und nur die für die Steuerzahlung erforderlichen Beträge entnimmt. In den Perioden 1 bis $\mathrm{n}$ ist für den Unternehmer dasselbe Verhalten optimal wie bei der oben analysierten Selbstfinanzierung, weil sein einziges Entscheidungsproblem - den Einbehalt der Periodengewinne vorausgesetzt - darin besteht, den Begünstigungsantrag zu stellen oder nicht zu stellen. Der zusätzliche Summand 1-N rührt daher, dass der Aufzinsungsfaktor $\Omega$ die Nachversteuerung des begünstigt versteuerten Gewinns aus Periode 0

18 Vgl. Homburg (2007b), S. 252. 
einschließt, vorliegend aber kein Gewinn begünstigt versteuert, sondern eine Einlage getätigt wurde, die am Anfang der Periode $n+1$ steuerfrei rückgewährt wird.

Ohne die Einlage würde das private Endvermögen auf $(1+\mathrm{A} \cdot \mathrm{i})^{\mathrm{n}}$ wachsen. Daher sind die Kapitalkosten der Beteiligungsfinanzierung $\mathrm{r}^{\mathrm{B}}$ implizit durch

$$
\Omega\left(r^{B}, n\right)+1-N=(1+A \cdot i)^{n}
$$

definiert, sofern die begünstigte Besteuerung mindestens einmal beantragt wird. Andernfalls betragen die Kapitalkosten der Beteiligungsfinanzierung $r^{B}=A / E \cdot i$, wie man durch Gleichsetzen der Endvermögen $\left(1+E \cdot r^{\mathrm{B}}\right)^{\mathrm{n}}$ und $(1+\mathrm{A} \cdot \mathrm{i})^{\mathrm{n}}$ sofort sieht. Dieser einfach gelagerte Fall sei zunächst vernachlässigt. Im Anhang des Beitrags wird gezeigt, dass der Aufzinsungsfaktor $\Omega$ streng monoton in der Rendite $r$ wächst. Somit liegen die Kapitalkosten der Beteiligungsfinanzierung, sofern der Antrag nach $\$$ 34a EStG-E gestellt wird, über den Kapitalkosten der Selbstfinanzierung, falls $\Omega\left(\mathrm{r}^{\mathrm{B}}, \mathrm{n}\right)>\Omega\left(\mathrm{r}^{\mathrm{S}}, \mathrm{n}\right)$. Aus (14) und (16) folgt

$$
\Omega\left(r^{B}, n\right)=\frac{B}{E} \cdot \Omega\left(r^{s}, n\right)-(1-N) .
$$

Diese Gleichung ergibt noch keinen Aufschluss, weil B/E größer als Eins ist und 1-N subtrahiert wird. Da aber die zeitweilige Antragsstellung das Endvermögen maximiert, gilt $\mathrm{B} \cdot \Omega\left(\mathrm{r}^{\mathrm{s}}, \mathrm{n}\right)>\mathrm{E} \cdot\left(1+\mathrm{E} \cdot \mathrm{r}^{\mathrm{s}}\right)^{\mathrm{n}}$. Aus $\mathrm{n} \geq \mathrm{a}(\mathrm{r})$ und $(10)$ folgt $(\mathrm{B}-\mathrm{E}) \cdot\left(1+\mathrm{E} \cdot \mathrm{r}^{\mathrm{s}}\right)^{\mathrm{n}}>\mathrm{B} \cdot(1-\mathrm{N})$. Durch Kombination dieser beiden Ungleichungen erhält man $(B-E) / E \cdot \Omega\left(r^{s}, n\right)>1-N$. Eingesetzt in (17) ergibt sich damit das Resultat

$$
\Omega\left(\mathrm{r}^{\mathrm{B}}, \mathrm{n}\right)>\frac{\mathrm{B}}{\mathrm{E}} \cdot \Omega\left(\mathrm{r}^{\mathrm{s}}, \mathrm{n}\right)-\frac{\mathrm{B}-\mathrm{E}}{\mathrm{E}} \cdot \Omega\left(\mathrm{r}^{\mathrm{s}}, \mathrm{n}\right)=\Omega\left(\mathrm{r}^{\mathrm{s}}, \mathrm{n}\right) .
$$

Demnach liegen die Kapitalkosten der Beteiligungsfinanzierung bei jedem endlichen Planungszeitraum über den Kapitalkosten der Selbstfinanzierung, sofern die Antragsstellung in mindestens einer Periode lohnt. Im Grenzübergang streben beide gegen denselben Wert A/B.i, wie man durch Potenzieren von (16) mit $1 / \mathrm{n}$ und Nutzung von (13) erkennt. Ist der Antrag in keiner Periode vorteilhaft, sind beide Kapitalkosten gleich A/E-i.

\section{Antragsstellung für den Gesamtgewinn}

Bis zu dieser Stelle wurde die Finanzierung eines Personenunternehmens der entsprechenden Finanzierung einer Kapitalgesellschaft nachgebildet, um Rechtsformvergleiche zu ermöglichen. Der Anteilseigner einer Kapitalgesellschaft, der Gewinne thesauriert oder das Stamm- bzw. Nennkapital anfangs erhöht, zahlt bis zum Anfang der Periode $n+1$ wegen des Trennungsprinzips keine Steuern aus seinem Privatvermögen. Aus Symmetriegründen wurde dasselbe für den (Einzel- bzw. Mit-) Unternehmer angenommen und unterstellt, dass dieser den Antrag auf begünstigte Besteuerung höchstens für den Gewinnanteil B stellt. Denn jeder darüber hinausgehende Antrag erfordert, wie eingangs dargelegt, $\mathrm{Zu}$ schüsse aus dem Privatvermögen. 
Obwohl diese Modellierung für Rechtsformvergleiche völlig befriedigend ist, lässt sie doch die Frage offen, ob ein Unternehmer, der die Rechtsform aus steuerlichen oder außersteuerlichen Gründen nicht wechseln möchte, von einer begünstigten Versteuerung des Gesamtgewinns profitieren kann. Diese Frage sei nun analysiert. Anders als bisher sind die Antragspolitiken des Unternehmers nicht mehr auf Zahlen aus dem Intervall [0; B] beschränkt, sondern sie dürfen jeden Wert aus dem Intervall $[0 ; 1]$ annehmen.

Beantragt der Unternehmer in Periode 0 die begünstigte Besteuerung des Gesamtgewinns, statt des Bruchteils B, steigt das betriebliche Endvermögen um (1-B). $\Omega(\mathrm{r}, \mathrm{n})$, weil die anfängliche Rücklage Eins statt B beträgt. Der Antrag erfordert eine Steuerzahlung in Höhe von $s^{\mathrm{b}}+s^{\mathrm{g}}$, nämlich die Zahlung der begünstigten Steuer und der nicht anrechenbaren Gewerbesteuer, aus dem Privatvermögen. Gemäß (6) und (7) lässt sich die Steuerzahlung als $s^{\mathrm{b}}+s^{\mathrm{g}}=\mathrm{E} / \mathrm{B} \cdot(1-\mathrm{B})$ schreiben. Die daraus resultierende Minderung des privaten Endvermögens beträgt E/B.(1-B) $\cdot(1+\mathrm{A} \cdot \mathrm{i})^{\mathrm{n}}$. Als Saldo des betrieblichen Vermögenszuwachses $(1-B) \cdot \Omega(r, s)$ und der privaten Vermögensminderung erhält man damit

$$
\frac{1-\mathrm{B}}{\mathrm{B}} \cdot\left[\mathrm{B} \cdot \Omega(\mathrm{r}, \mathrm{n})-\mathrm{E} \cdot(1+\mathrm{A} \cdot \mathrm{i})^{\mathrm{n}}\right] .
$$

Ein Blick auf die Definition (14) zeigt: Die Antragsstellung für den Gesamtgewinn statt für den Gewinnbruchteil B erbringt keinen Vorteil, wenn die Unternehmensrendite den Kapitalkosten der Selbstfinanzierung entspricht. Dies lässt sich wie folgt erklären: Bei einer Unternehmensrendite in Höhe der Kapitalkosten der Selbstfinanzierung ist der Unternehmer in Periode 0 indifferent, ob er Gewinnanteile einbehalten oder entnehmen soll, weil einbehaltene Gewinne nach Steuern dasselbe Endvermögen ergeben wie entnommene Gewinne. Die Antragsstellung für den Gesamtgewinn bei Zahlung der betrieblich veranlassten Steuern aus dem Privatvermögen ist nichts anderes als eine geringere Entnahme und damit eine Selbstfinanzierung sui generis. Da Einlagen und Entnahmen keinen Einfluss auf das Endvermögen haben, gilt dasselbe für die Antragsstellung; es ist gleichgültig, ob der Unternehmer die begünstigte Besteuerung für den Gesamtgewinn oder den Bruchteil B beantragt, sofern er im letzteren Fall den Gewinnanteil, der zur Steuerzahlung benötigt wird, entnimmt. Aus diesem Grund ist die Beschränkung des Begünstigungsantrags auf den Bruchteil B nicht bloß vordergründig realistisch, sondern für ein reifes Unternehmen, das die Rendite durch seine Gewinnverwendungspolitik auf die Kapitalkosten der Selbstfinanzierung gedrückt hat, auch normativ richtig.

Anders verhält es sich für junge Unternehmen, die auf Fremdfinanzierung verzichten müssen oder wollen und daher auf Beteiligungsfinanzierung angewiesen sind. Nach gängiger Lehre sollte ein Unternehmer unter dieser Voraussetzung so lange Einlagen tätigen, wie die Unternehmensrendite über den Kapitalkosten der Beteiligungsfinanzierung liegt. $\mathrm{Ab}$ diesem Punkt erbringen zusätzliche Einlagen keinen Vorteil, wohl aber „virtuelle Einlagen", die darin bestehen, dass der Unternehmer die begünstigte Besteuerung des Gesamtgewinns beantragt und die Steuerzahlungen aus dem Privatvermögen leistet. Denn laut (19) lohnt die begünstigte Versteuerung des Gesamtgewinns so lange, wie die Unter- 
nehmensrendite über den Kapitalkosten der Selbstfinanzierung liegt, und die letzteren sind stets geringer als die Kapitalkosten der Beteiligungsfinanzierung.

Die begünstigte Versteuerung des Gesamtgewinns ist demnach vorteilhaft, wenn die Unternehmensrendite zwischen den Kapitalkosten der Selbstfinanzierung und der Beteiligungsfinanzierung liegt, wenn also $\mathrm{r}^{\mathrm{B}}>\mathrm{r}>\mathrm{r}^{\mathrm{s}}$ gilt. Liegt die Unternehmensrendite unter den Kapitalkosten der Selbstfinanzierung, dann verringert die begünstigte Versteuerung des Gesamtgewinns das Vermögen. Übersteigt die Unternehmensrendite aber sogar die Kapitalkosten der Beteiligungsfinanzierung, sollte Privatvermögen sofort ins Unternehmen eingelegt werden, statt es für spätere Steuerzahlungen zurückzuhalten. Weil die Kapitalkosten der Selbstfinanzierung und der Beteiligungsfinanzierung bei kurzem Planungshorizont $\mathrm{n}<\mathrm{a}(\mathrm{r})$ identisch sind und langfristig gegen denselben Wert streben, ist der Korridor für die Vorteilhaftigkeit einer begünstigten Versteuerung des Gesamtgewinns allerdings schmal.

\section{Beispiele}

Abschließend sei die obige Analyse durch einige konkrete Beispiele illustriert, die ein Gefühl für die fraglichen Größenordnungen vermitteln. Betrachtet wird ein konfessionsloser Steuerpflichtiger, dessen Tätigkeit einem Gewerbesteuerhebesatz von 400 \% unterliegt.

\begin{tabular}{|c|c|c|c|c|}
\hline $\mathbf{s}^{\text {tarif }}$ & $\mathbf{r}$ & $5 \%$ & $10 \%$ & $20 \%$ \\
\hline & & 300 & 155 & 83 \\
\hline & & 56 & 29 & 16 \\
\hline & & 11 & 6 & 3 \\
\hline & & 2 & 1 & 1 \\
\hline
\end{tabular}

Tabelle 1. Konstante Antragspolitik (Jahre).

Die in Tabelle 1 aufgeführten Zahlen zeigen, ab welchem Planungszeitraum die ständige Antragsstellung gegenüber dem ständigen Antragsverzicht vorteilhaft ist. Offenbar wird die Antragsstellung mit steigender Unternehmensrendite tendenziell früher vorteilhaft. Höhere Tarifbelastungen begünstigen die Antragsstellung ebenfalls, wobei der starke Einfluss der Tarifbelastung in der Region zwischen dem bisherigen Spitzensteuersatz (42 \%) und seinem Nachfolger ( $45 \%$ ) auffällt: Während der Vorteil der begünstigten Versteuerung beim bisherigen Spitzensteuersatz oft fraglich erscheint, lohnt sich die Antragsstellung für Unternehmer, die dem neuen Spitzensteuersatz unterliegen, auch im Fall kurzer Planungszeiträume. Es scheint, als habe der Gesetzgeber die Parameter nicht im Hinblick auf die angeblich erstrebte - und weit verfehlte - Rechtsformneutralität gewählt, sondern jene Unternehmer teilweise entschädigen wollen, die ab 2008 „Reichensteuer“ zahlen. 


\begin{tabular}{|c|c|c|c|c|}
\hline $\boldsymbol{s}^{\text {tarif }}$ & $\mathbf{r}$ & $\mathbf{5 \%}$ & $\mathbf{1 0} \%$ & $\mathbf{2 0} \%$ \\
\hline $30 \%$ & 69 & 35 & 18 \\
\hline $35 \%$ & 31 & 16 & 9 \\
\hline $42 \%$ & 9 & 5 & 3 \\
\hline $45 \%$ & 2 & 1 & 1 \\
\hline
\end{tabular}

Tabelle 2. Antragsverzichtszeiträume (Jahre).

Tabelle 2 weist die Antragsverzichtszeiträume a(r) aus. Deren Muster stimmt qualitativ mit dem aus Tabelle 1 überein, doch sind die Zahlen durchweg kleiner, was die Wichtigkeit der Unterscheidung konstanter und gemischter Antragspolitiken unterstreicht. Beispielhaft sei ein Unternehmer betrachtet, der einer Tarifbelastung von $35 \%$ unterliegt und eine Unternehmensrendite von $10 \%$ erwirtschaftet. Als Planungshorizont sei n=20 angenommen. Nach der Angabe in Tabelle 2 erreicht der Unternehmer das höchste Endvermögen, wenn er den Antrag auf begünstigte Besteuerung in den Jahren 0 bis 4 stellt und in den Jahren 5 bis 20 nicht stellt. Demgegenüber wäre die konstante Antragsstellung ausweislich Tabelle 1 erst ab einem Planungshorizont von 29 Jahren vorteilhaft.

\begin{tabular}{|c|c|c|c|c|}
\hline $\mathbf{s}^{\text {tarif }}$ & $\mathbf{n}$ & $\mathbf{5}$ & $\mathbf{1 0}$ & $\boldsymbol{1}$ \\
\hline \multirow{2}{*}{$30 \%$} & $4,3 \%$ & $4,3 \%$ & $4,2 \%$ \\
& $(4,3 \%)$ & $(4,3 \%)$ & $(4,2 \%)$ \\
\hline $35 \%$ & $4,7 \%$ & $4,7 \%$ & $4,3 \%$ \\
& $(4,7 \%)$ & $(4,7 \%)$ & $(4,3 \%)$ \\
\hline $42 \%$ & $5,3 \%$ & $5,2 \%$ & $4,5 \%$ \\
& $(5,3 \%)$ & $(5,3 \%)$ & $(4,5 \%)$ \\
\hline $45 \%$ & $4,9 \%$ & $4,8 \%$ & $4,6 \%$ \\
& $(5,6 \%)$ & $(5,5 \%)$ & $(4,6 \%)$ \\
\hline
\end{tabular}

Tabelle 3. Kapitalkosten der Selbstfinanzierung. (In Klammern: Kapitalkosten der Beteiligungsfinanzierung).

Schließlich illustriert Tabelle 3 die Kapitalkosten bei optimaler Antragspolitik. Die Werte beruhen auf den bisherigen Annahmen und einem Zinssatz von $4 \%$. Bei wachsendem Planungszeitraum nehmen die Kapitalkosten erwartungsgemäß ab, weil die Nachversteuerung immer weniger ins Gewicht fällt. Hingegen mag der Einfluss des tariflichen Steuersatzes auf die Kapitalkosten zunächst überraschen, denn erstens ist die Wirkung nicht monoton und zweitens nehmen die asymptotischen Kapitalkosten bei steigendem Steuersatz zu. Im Fall einer hohen Tarifbelastung ist die begünstigte Besteuerung zwar tendenziell vorteilhafter im Sinne einer früheren Antragsstellung, doch schneidet sie gemessen an den Kapitalkosten schlechter ab. Die Erklärung für diesen scheinbaren Widerspruch liegt darin, dass die Kapitalkosten den Vorteil des Antrags auf begünstigte Besteuerung relativ zur Entnahme messen und nicht relativ zum Unterlassen des Antrags. Nach Formel (15) entsprechen die asymptotischen Kapitalkosten dem Ausdruck A/B.i, und weil die Abgeltungsteuer von den persönlichen Verhältnissen unabhängig ist, der Steuerfaktor B bei zunehmender Tarifbelastung aber sinkt, implizieren hohe Tarifbelastungen hohe Kapitalkosten. 
Aus diesem Blickwinkel ist die vorherrschende Ansicht, die begünstigte Besteuerung nach $\$ 34 a$ EStG-E sei vorwiegend für große Personenunternehmen geeignet, fragwürdig. Weitere Zweifel erwachsen daraus, dass die Kapitalkosten der Fremdfinanzierung ziemlich genau dem Zinssatz entsprechen, also weit niedriger sind, und die Kapitalkosten der Selbstfinanzierung einer Kapitalgesellschaft - als Alternative zum Personenunternehmen unter den hiesigen Annahmen nur 4,2 \% betragen. Nach den Angaben in Tabelle 3 können nur Steuerpflichtige mit sehr niedriger Tarifbelastung, aber nicht die Teilhaber großer Personengesellschaften, vergleichbar geringe Kapitalkosten erreichen.

\section{Schluss}

In diesem Beitrag wurde gefragt, inwieweit ein Einzel- oder Mitunternehmer, der den nach Steuern verbleibenden Gewinn einbehält oder Einlagen tätigt, die neue Vorschrift $\$ 34$ a EStG-E nutzen sollte. Es zeigte sich, dass die optimale Antragspolitik vom Planungshorizont, der Unternehmensrendite und der tariflichen Steuerbelastung abhängt. Vor allem aber gilt unabhängig vom Planungshorizont, dass eine Antragsstellung in jedem Jahr niemals optimal ist. Das höchste Endvermögen resultiert vielmehr, wenn der Unternehmer die begünstigte Besteuerung zunächst beantragt und später nicht mehr. Der optimale Umschaltzeitpunkt und die bei optimaler Antragspolitik entstehenden Kapitalkosten der Eigenfinanzierung wurden mathematisch beschrieben, wobei sich zeigte, dass die Kapitalkosten bei steigendem tariflichen Steuersatz zunehmen, weshalb die Eigenfinanzierung vor allem für Bezieher höherer Einkommen nachteilig ist. Schließlich wurde gezeigt, dass die Antragsstellung für den gesamten Gewinn, statt für den Bruchteil B, im Fall eines reifen Personenunternehmens, dessen Rendite mit den Kapitalkosten der Selbstfinanzierung übereinstimmt, keinen Vorteil bringt.

Mit den $\$ \$ 32 \mathrm{~d}$ und 34a EStG-E hat der Gesetzgeber die zuvor halbwegs gegebene Finanzierungsneutralität bei Personenunternehmen zerstört, die angestrebte Rechtsformneutralität grob verfehlt - weil einbehaltene Gewinne bei korrekter Rechnung erheblich stärker belastet werden als thesaurierte Gewinne - und ein System etabliert, dessen Belastungswirkungen weder die Steuerpflichtigen noch das Gros ihrer Berater durchschauen können. Wahrscheinlich müssen die heutigen Kompetenzzentren für „corporate finance“ künftig durch Zentren für „partnership finance“ ergänzt werden.

\section{Literaturangaben}

Förster (2007): Anrechnung der Gewerbesteuer auf die Einkommensteuer nach der Unternehmensteuerreform 2008, Der Betrieb, S. 760-764.

Harberger (1962): The Incidence of the Corporation Income Tax, Journal of Political Economy, S. 215-240.

Homburg (2007a): Die Abgeltungsteuer als Instrument der Unternehmensfinanzierung. Deutsches Steuerrecht, S. 686-692. 
Homburg (2007b): Allgemeine Steuerlehre, 5. Auflage, München.

Homburg/Houben/Maiterth (2007): Rechtsform und Finanzierung nach der Unternehmensteuerreform 2008, Die Wirtschaftsprüfung, S. 376-381.

Kaminski/Hofmann/Kaminskaite (2007): Erste Überlegungen zur Rechtsformwahl nach dem Entwurf zur Unternehmensteuerreform 2008, Die Steuerberatung, S. 161168.

Kessler/Ortmann-Babel/Zipfel (2007): Unternehmensteuerreform 2008: Die geplanten Änderungen im Überblick, Betriebs-Berater, S. 523-534.

Kiesewetter/Lachmund (2004): Wirkungen einer Abgeltungssteuer auf Investitionsentscheidungen und Kapitalstruktur von Unternehmen, Die Betriebswirtschaft, S. 395-411.

Kiesewetter/Niemann (2004): Steuerparadoxa durch Endbesteuerung, Mindestbesteuerung und Begünstigung einbehaltener Gewinne, Journal für Betriebswirtschaft, S. 129-139.

Kleineidam/Liebchen (2007): Die Mär von der Steuerentlastung durch die Unternehmensteuerreform 2008, Der Betrieb, S. 409-412.

König (1997): Ungelöste Probleme einer investitionsneutralen Besteuerung - Gemeinsame Wurzel unterschiedlicher neutraler Steuersysteme und die Berücksichtigung unsicherer Erwartungen, Zeitschrift für betriebswirtschaftliche Forschung, S. 42-63.

Kruschwitz/Löfler (2006): Discounted cash flow: a theory of the valuation of firms, Chichester.

Kruschwitz (2007): Investitionsrechnung, 11. Auflage, Oldenburg.

Löffler/Schneider (2003): Martingales, Taxes, and Neutrality, Diskussionspapier Nr. 269, Wirtschaftswissenschaftliche Fakultät, Universität Hannover.

Maiterth/Sureth (2006): Unternehmensfinanzierung, Unternehmensrechtsform und Besteuerung, Betriebswirtschaftliche Forschung und Praxis, S. 225-245.

Niemann (2001): Neutrale Steuersysteme unter Unsicherheit - Besteuerung und Realoptionen, Bielefeld.

Sachverständigenrat (2006): Reform der Einkommens- und Unternehmensbesteuerung durch die Duale Einkommensteuer, Wiesbaden.

Schreiber (2002): Die Steuerbelastung der Personenunternehmen und der Kapitalgesellschaften - Ein Beitrag zur Weiterentwicklung der Unternehmensbesteuerung, Die Wirtschaftsprüfung, S. 557-571.

Sinn (1990): Taxation and the Cost of Capital: The 'Old' View, the 'New' View, and Another View, in: D. Bradford, ed., Tax Policy and the Economy 5, 1991, National Bureau of Economic Research, pp. 25-54. 
Sorensen (1995): Changing Views of the Corporate Income Tax, National Tax Journal, S. 279-294.

Spengel (2006): Besteuerung von Einkommen - Aufgaben, Wirkungen und europäische Herausforderungen, Gutachten G zum 66. Deutschen Juristentag Stuttgart 2006, München.

Sureth (1999): Der Einfluss von Steuern auf Investitionsentscheidungen bei Unsicherheit, Wiesbaden.

\section{Anhang: Beweis der strengen Monotonie von $\Omega$ in $\mathbf{r}$}

Der Beweis kann nicht durch Ableiten geführt werden, weil a(r) als fallende Treppenfunktion nicht differenzierbar ist. Man definiere die Hilfsgröße

$$
\mathrm{Z}(\mathrm{r}, \mathrm{n})=(1+\mathrm{B} \cdot \mathrm{r})^{\mathrm{n}-\mathrm{a}\left(\mathrm{r}_{0}\right)} \cdot\left[(1+\mathrm{E} \cdot \mathrm{r})^{\mathrm{a}\left(\mathrm{r}_{0}\right)}-(1-\mathrm{N})\right] .
$$

$\mathrm{Z}(\mathrm{r}, \mathrm{n})$ ist jene hypothetische Verzinsung, die sich ergibt, wenn der Antragsverzichtszeitraum bei gegebener Unternehmensrendite $r$ nicht optimal gewählt, sondern auf dem $\mathrm{Ni}$ veau $\mathrm{a}\left(\mathrm{r}_{0}\right)$ fixiert wird. Weil a $(\mathrm{r})$ optimal ist und $\mathrm{a}\left(\mathrm{r}_{0}\right)$ möglicherweise suboptimal, gilt

$$
\Omega(r, n) \geq Z(r, n) .
$$

Man betrachte nun ein $r>r_{0}$. Aus (10) folgt $(1+E \cdot r)^{a(r)}-(1-N)>0$, und wegen $a\left(r_{0}\right) \geq a(r)$ ist der eckig geklammerte Ausdruck in (20) erst recht strikt positiv. Daher wächst $Z(r, n)$ offensichtlich streng monoton in $\mathrm{r}$ :

$$
\mathrm{Z}(\mathrm{r}, \mathrm{n})>\mathrm{Z}\left(\mathrm{r}_{0}, \mathrm{n}\right) .
$$

Schließlich folgt aus den Definitionen (12) und (20)

$$
\mathrm{Z}\left(\mathrm{r}_{0}, \mathrm{n}\right)=\Omega\left(\mathrm{r}_{0}, \mathrm{n}\right) .
$$

Durch Verkettung der drei vorigen Ungleichungen erhält man $\Omega(\mathrm{r}, \mathrm{n})>\Omega\left(\mathrm{r}_{0}, \mathrm{n}\right)$ für alle $r>r_{0}$ und damit die behauptete strenge Monotonie von $\Omega$ in $r$.

q.e.d. 